\title{
Distribuição e características dos programas de Mestrado Profissional em ensino em saúde no Brasil
}

\author{
Distribution and characteristics of the masters professional programs in health education in \\ Brazil
}

Distribución y características de los programas de Maestrías Profesionales en enseñanza en salud en Brasil

Sarah Lais Rocha ${ }^{1,2 *}$, Edna Ferreira Coelho Galvão ${ }^{3}$, David José Oliveira Tozetto ${ }^{1}$, Maria Alice Alves Fernandes $^{1}$, Robson José de Souza Domingues ${ }^{3}$.

\section{RESUMO}

Objetivo: Buscou-se verificar a distribuição e características dos Mestrados Profissionais (MP) em Ensino em Saúde no Brasil, bem como, agrupar por regiões a disponibilidade de Mestrados Profissionais em Ensino em Saúde no Brasil. Métodos: Para alcançar o objetivo da pesquisa, optou-se pela realização de um estudo exploratório, retrospectivo, com abordagem quantitativa. Isto foi possível por meio da investigação na aba Coleta Capes sobre Dados Cadastrais dos Programas. Os dados obtidos foram analisados por estatística descritiva com auxílio do software IBM SPSS Statistics. Resultados: Foram coletados dados de 13 Programas de Pós-Graduação - PPG. Destes, 8 (61,5\%) são instituições públicas e 5 (38,4\%) instituições privadas. Grande maioria (84,6\%) tem nota 3 de avaliação. Em relação a distribuição espacial no país é bastante uniforme sendo que Norte, Sul e Centro-Oeste comportam cada região 2 Programas de Mestrado Profissional em Ensino em Saúde, Sudeste e Nordeste 3 PPG. Conclusão: Apesar de muitos avanços na criação de novos programas de MP para formação dos docentes na área da saúde, ainda se encontra distante de atingir um patamar ideal.

Palavras-Chave: Mestrado Profissional, Ensino em Saúde, Ensino de Pós-graduação.

\begin{abstract}
Objective: It was sought to understand the Professional Master's Degree in Health Education in Brazil, as well as group by regions the availability of Professional Masters in Education in Health in Brazil. Methods: In order to reach the research objective, an exploratory, retrospective study was conducted with quantitative measures. This was possible through the survey in the Collection Capes tab on the Cadastral Data of the Programs The data were scaled by descriptive statistics with the aid of the software IBM SPSS Statistics. Results: It were collected data from 13 Post graduate Programs - PPG. Of these, 8 (61.5\%) are public institutions and $5(38.4 \%)$ are privateinstitutions. The vast majority (84.6\%) have a rating of 3 . In relation to the spatial distribution in the country it is very uniform, and the North, South and Center-West contains each region 2 Professional Master's Programs in Teaching in Health, Sudente and Northeast 3 PPG. Conclusion: Despite many advances in the creation of new MP programs for teacher training in health, it is still far from reaching an ideal level.
\end{abstract}

Key words: Professional Master's Degree, Health Teaching, Post graduate teaching.

${ }^{1}$ Professor(a) do Curso de Graduação em Medicina, Universidade do Estado do Pará/UEPA, Marabá, Pará, Brasil *E-mail:sarahlaisrocha@gmail.com

${ }^{2}$ Professor(a) do Curso de Graduação em Enfermagem da Faculdade Carajás, Marabá, Pará.

${ }^{3}$ Professor(a) orientador(a) Doutor(a) do PPG Mestrado Profissional em Ensino em Saúde na Amazônia, Universidade do Estado do Pará/UEPA, Belém, Pará, Brasil.

${ }^{4}$ Discente do Curso de Graduação em Medicina, Universidade do Estado do Pará/UEPA, Marabá, Pará. 


\section{RESUMEN}

Objetivo: Se buscó entender la distribución y características de los Maestría Profesionales en Enseñanza en Salud en Brasil, así como, agrupar por regiones la disponibilidad de Maestros Profesionales en Enseñanza en Salud en Brasil. Métodos: Para alcanzar el objetivo de la investigación, se optó por la realización de un estudio exploratorio, retrospectivo, con abordaje cuantitativo. Esto fue posible por medio de la investigación en la pestaña Recolección Capes sobre Datos Catastrales de los Programas. Los datos obtenidos fueron analizados por estadística descriptiva con ayuda del software IBM SPSS Statistics. Resultados: Fueron recogidos datos de 13 Programas de Postgrado - PPG. De estos, 8 (61,5\%) soninstituciones públicas y 5 $(38,4 \%)$ instituciones privadas. La mayoría $(84,6 \%)$ tiene una nota 3 de evaluación. Encuanto a ladistribución espacial enel país es bastante uniforme siendo que Norte, Sur y Centro-Oeste comporta cada región 2 Programas de Maestría Profesional em Enseñanza em Salud, Sudente y Nordeste 3 PPG. Conclusión: A pesar de muchos avances enlacreación de nuevos programas de MP para la formación de los docentes em el área de la salud, todavía se encuentra distante de alcanzar um nivel ideal.

Palabrasclave: Maestría Profesional, Enseñanza en Salud, Enseñanza de Postgrado.

\section{INTRODUÇÃO}

O início do desenvolvimento da pós-graduação brasileira foi orientado pelo Conselho Federal de Educação (CFE), que definiu e fixou as características dos Programas de pós-graduação (PPG) Stricto sensu no Brasil em dezembro de 1965, com a aprovação do Parecer n 977 (BRASIL, 1965). Neste parecer, a defesa de se instituir esse nível de formação se alicerçava na necessidade de assegurar treinamento eficaz à técnicos e trabalhadores para atuarem na transferência de conhecimento; a princípio a pós-graduação era considerada uma forma de qualificar profissionais para o mercado de trabalho.

A partir deste Parecer $n^{\circ}$ 977/1965, também conhecido como Parecer Sucupira até a década de 90 era possível identificar apenas a modalidade de Mestrado Acadêmico (MA), mais tarde, foram criados os Mestrados Profissionais (MP), essas duas modalidades podem ser diferenciadas pelos seus objetivos centrais - o Mestrado acadêmico e o Mestrado profissional. Enquanto os programas acadêmicos ocupam-se de questões relativas à aprendizagem, à epistemologia, à resolução de problemas, à História e à Filosofia da Ciência, os programas profissionais estão voltados diretamente para capacitar profissionais para o exercício da prática profissional avançada e transformadora de procedimentos, visando atender demandas sociais, organizacionais ou profissionais e do mercado de trabalho (BRASIL, 1965).

O Mestrado Profissional nasce em 1995, porém, sua existência legal decorre de uma Portaria da Capes $n^{\circ} 80$, baixada em novembro de 1998, seguindo portarias e resoluções que tentam estabelecer as diferenças entre os cursos acadêmicos e profissionais. Com formato e denominação polêmicos o MP esbarra na concepção acadêmica da pós-graduação, inclusive a validade deste título foi assunto foi de discussão pelo Conselho Nacional de Educação, e ficou bastante claro que, numa seleção esse critério não pode ser exigido especificamente (RIBEIRO RJ, 2006).

Mais recentemente, a Portaria no 389, de março de 2017, do Ministério da Educação atualizou a legislação que dispõe sobre os Programas de pós-graduação (PPG) profissional, incluindo o doutorado profissional, no âmbito do PPG stricto sensu, já que até então apenas a categoria Mestrado era possível na formação profissional. Essa portaria traz, em seu Art. $2^{\circ}$, que o aluno em formação deve transferir conhecimento para a sociedade, atendendo demandas específicas e de arranjos produtivos, com vistas o desenvolvimento nacional, regional ou local.

Os Mestrados Profissionais - MPs, foram pensados com o objetivo de qualificar os profissionais para atuar como formadores e indutores de processos de mudança em seus espaços de trabalho mediante a adoção de novos conceitos e práticas, e gerar produtos que devem tenham alta aplicabilidade (HORTALE et al, 2010). A necessidade deste produto é regulamentada pela Coordenação de Aperfeiçoamento de Pessoal de Nível

REAS/EJCH |Vol.11 (10) | e872 |DOI: https://doi.org/10.25248/reas.e872.2019 Página 2 de 6 
Superior - Capes, esse órgão destaca que a pesquisa proposta em um Mestrado Profissional deve gerar "[...] aplicativos, materiais didáticos ou instrucionais, produtos, processos ou técnicas" (BRASIL, 2009, p. 3).

A Área de Ensino integra a Grande Área Multidisciplinar. Foi constituída pela Portaria Capes n83/2011. Ela foi nucleada na antiga Área de Ensino de Ciências e Matemática, da qual guarda as principais referências e experiência de organização e avaliação de PPG, justificando-se a sua criação dos pontos de vista epistemológico, educacional e social (BRASIL, 2016).

Neste sentido, o propósito deste estudo foi: verificar a distribuição e características dos MP em Ensino em Saúde no Brasil, e de forma mais específica, agrupar por regiões a disponibilidade de Mestrados Profissionais em Ensino em Saúde no Brasil. Considera-se também que entender o desenvolvimento dos Programas de Mestrado Profissional na área de Ensino em Saúde irá despertar o estímulo para mais pesquisas relacionadas ao tema, cooperando para a qualidade e consolidação dos Cursos de Mestrado Profissional em Ensino em Saúde, principalmente no interior da Amazônia, este contexto justifica a realização deste estudo.

\section{MÉTODOS}

Optou-se pela realização de um estudo exploratório com base numa análise documental, retrospectivo, com abordagem de natureza quantitativa. Para o desenvolvimento da pesquisa foram selecionados os PPGs em Ensino em Saúde no Brasil, com a finalidade de estudar uma amostra nacional. A investigação ocorreu na aba Coleta Capes sobre Dados Cadastrais do Programa, usando-se como filtros: área de avaliação: ensino; modalidade: Mestrado Profissional.

Foram encontrados 124 registros, dos quais foram selecionados os da área de Ensino na Saúde e, posteriormente, confrontados com o Relatório de Avaliação da Área de Ensino. Foram excluídos da amostra os Mestrados que estavam desativados ou em desativação.

O período foi selecionado para o estudo foi de janeiro entre 2013 a dezembro de 2016, por ser uma fase de avaliação considerada para a Capes, que faz as avaliações de forma quadrienal. Os dados do estudo são de domínio público e de acesso irrestrito.

As informações foram inseridas numa planilha eletrônica elaborada pela pesquisadora, onde foram registradas as informações em partes: com características da instituição e do Programa de Pós-Graduação, para a análise dos dados, as variáveis categóricas foram representadas por meio de frequências e porcentagens (RAZALI NM, WAH YB, 2011). As análises foram conduzidas com o software IBM Statistical Package for the Social Sciences (SPSS $\AA$, Chicago, IL, EUA) 20.0.

\section{RESULTADOS E DISCUSSÃO}

Os resultados encontrados nesse estudo delineiam um diagnóstico da realidade dos Mestrados Profissionais em Ensino em Saúde no Brasil. No Quadro 1 é possível observar as características dos PPGs na modalidade MP segundo nome do programa, sigla da instituição, ano de recomendação, nota de avaliação do curso, bem como estado e região onde está localizado o programa. Em pesquisas futuras de maior fôlego, temos a pretensão de aprofundar o diagnóstico no sentido de evidenciar as áreas de pesquisa contempladas e os impactos nas regiões brasileiras.

Visualizar diferentes programas presentes em todas as regiões do território nacional provoca, um sentimento de justiça de acesso das conquistas dessa modalidade de PPG. Esse diagnóstico reforça, o papel de cada programa que, nesse contexto, tornam-se indispensáveis para potencializar a formação de professores para o ensino em saúde no Brasil (Quadro 1).

O que se espera de um Mestrado Profissional, que os profissionais, a partir da pesquisa, possam transformar o contexto social e profissional. Ou seja, objetiva-se formar um profissional que atuando num mundo "externo à academia, saiba localizar, reconhecer e identificar, e, sobretudo, utilizar a pesquisa de modo que agregue valor a suas atividades, sejam essas de interesse mais pessoal ou mais social (GOUVÊA MV, et al, 2016). 
Quadro 1 - Programas de pós-graduação na modalidade Mestrado Profissional em Ensino em Saúde no Brasil no período entre 2013 e 2016.

\begin{tabular}{|c|c|c|c|c|c|}
\hline Programa / Instituição & Sigla da Instituição & Ano & Nota & UF & Região \\
\hline $\begin{array}{l}\text { ENSINO EM SAÚDE } \\
\text { Centro Universitário Christus }\end{array}$ & UNICHRISTUS & 2015 & 3 & CE & NORDESTE \\
\hline $\begin{array}{l}\text { ENSINO EM CIÊNCIAS DA SAÚDE E } \\
\text { DO MEIO AMBIENTE } \\
\text { Centro Universitário de Volta } \\
\text { Redonda }\end{array}$ & UNIFOA & 2007 & 4 & RJ & SUDESTE \\
\hline $\begin{array}{l}\text { ENSINO EM SAÚDE } \\
\text { Centro Universitário do Estado do } \\
\text { Pará }\end{array}$ & CESUPA & 2015 & 3 & PA & NORTE \\
\hline $\begin{array}{l}\text { EDUCAÇÃO PARA O ENSINO NA } \\
\text { ÁREA DE SAÚDE } \\
\text { Faculdade Pernambucana de Saúde }\end{array}$ & FPS & 2010 & 3 & PE & NORDESTE \\
\hline $\begin{array}{l}\text { ENSINO NA SAÚDE } \\
\text { Fundação Univ. Federal de Ciências } \\
\text { da Saúde de Porto Alegre }\end{array}$ & UFCSPA & 2013 & 3 & RS & SUL \\
\hline $\begin{array}{l}\text { SAÚDE E EDUCAÇÃO } \\
\text { Universidade de Ribeirão Preto }\end{array}$ & UNAERP & 2011 & 3 & SP & SUDESTE \\
\hline $\begin{array}{l}\text { FORMAÇÃO INTERDISCIPLINAR } \\
\text { EM SAÚDE } \\
\text { Universidade de São Paulo }\end{array}$ & USP & 2013 & 3 & SP & SUDESTE \\
\hline $\begin{array}{l}\text { ENSINO EM SAÚDE NA AMAZÔNIA } \\
\text { Universidade do Estado do Pará }\end{array}$ & UEPA & 2011 & 3 & PA & NORTE \\
\hline $\begin{array}{l}\text { ENSINO EM SAÚDE } \\
\text { Universidade Estadual de Mato } \\
\text { Grosso do Sul }\end{array}$ & UEMS & 2013 & 3 & MS & $\begin{array}{l}\text { CENTRO- } \\
\text { OESTE }\end{array}$ \\
\hline $\begin{array}{l}\text { ENSINO NA SAÚDE } \\
\text { Universidade Federal de Alagoas }\end{array}$ & UFAL & 2010 & 3 & $\mathrm{AL}$ & NORDESTE \\
\hline $\begin{array}{l}\text { ENSINO NA SAÚDE } \\
\text { Universidade Federal de Goiás }\end{array}$ & UFG & 2010 & 3 & GO & $\begin{array}{l}\text { CENTRO- } \\
\text { OESTE }\end{array}$ \\
\hline $\begin{array}{l}\text { ENSINO EM CIÊNCIAS DA SAÚDE } \\
\text { Universidade Federal de São Paulo }\end{array}$ & UNIFESP & 2002 & 4 & SP & SUDESTE \\
\hline $\begin{array}{l}\text { ENSINO NA SAÚDE } \\
\text { Universidade Federal do Rio Grande } \\
\text { do Norte }\end{array}$ & UFRN & 2012 & 3 & $\mathrm{RN}$ & NORDESTE \\
\hline
\end{tabular}

Fonte: Dados da pesquisa, 2019.

Foram encontrados 13 Programas de pós-graduação - PPG na modalidade Mestrado Profissional em Ensino em Saúde no Brasil. Destes, 8 representando 61,5\% da amostra são instituições públicas e 5 perfazendo $38,4 \%$ são de instituições privadas. A expressiva maioria com $84,6 \%$ tem nota 3 de avaliação. Em relação a distribuição espacial no país é bastante equânime sendo que Norte, Sul e Centro-Oeste comportam cada região 2 Programas de Mestrado Profissional em Ensino em Saúde e Sudente e Nordeste 3 PPG (Tabela 1). Essa característica de distribuição também foi encontrada por Barbosa $V$ (2016) que pesquisou sobre os MP em educação nas Universidades Brasileiras. 
Tabela 1 - Programas de pós-graduação na modalidade Mestrado Profissional em Ensino em Saúde no Brasil segundo classificação administrativa, região e nota de avaliação no período entre 2013 e 2016.

\begin{tabular}{lc}
\hline Características & Geral $\mathbf{( N = 1 3 )}$ \\
\hline Classificação Administrativa & \\
$\quad$ Instituição pública & $8(61,5)$ \\
Instituição privada & $5(38,4)$ \\
Região & $2(15,4)$ \\
$\quad$ Centro Oeste & $4(30,8)$ \\
Nordeste & $2(15,4)$ \\
Norte & $4(30,8)$ \\
Sudeste & $1(7,7)$ \\
Sul & \\
Nota do curso de mestrado & $11(84,6)$ \\
3 & $2(15,4)$ \\
4 &
\end{tabular}

Os dados são apresentados como n (\%).

Fonte: Dados da pesquisa, 2019.

Apesar de muitos avanços a formação dos docentes na área da saúde, ainda se encontra distante de atingir um patamar ideal. No Brasil, a grande maioria dos docentes dos cursos da área da saúde carece de uma formação pedagógica. Percebe-se, uma tendência a reduzir os saberes dos professores a aspectos técnico-científicos, empobrecendo as possibilidades de transformação e avanço nas relações de aprendizagem e ensino (BRANDÃO RB, BATISTA NA 2016).

Gráfico 1 - Evolução da implantação dos MPs em Ensino em Saúde no Brasil até o ano de 2016.

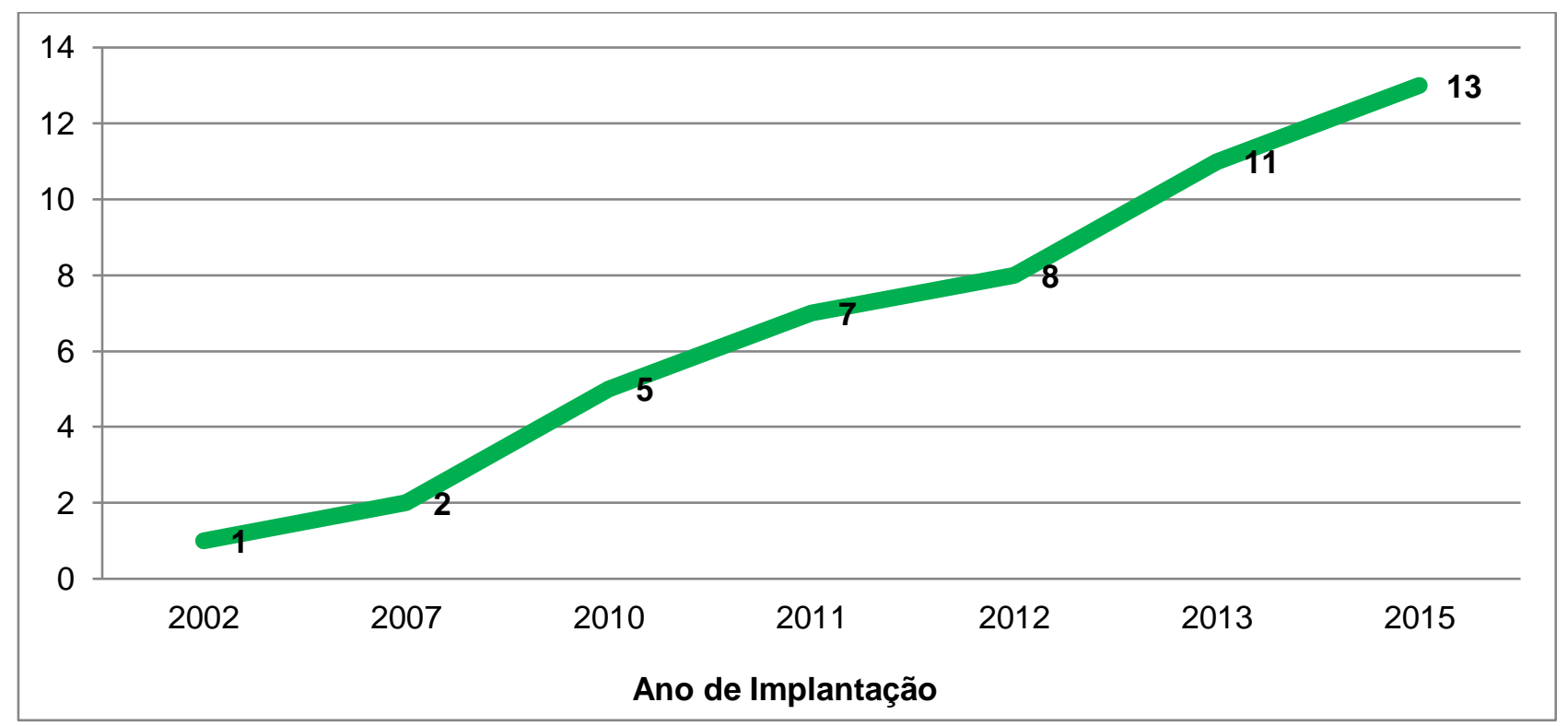

Fonte: Dados da pesquisa, 2019.

Em relação a implantação dos MPs em Ensino em Saúde no Brasil, percebe-se um aumento importante desse tipo de PPG após o ano de 2010, sendo que antes disso só existiam 16\% dos cursos implantados. Segundo a Capes, no Documento de Avaliação da Área de Ensino (BRASIL, 2016), o número de egressos em Mestrados Profissionais no triênio 2004-2006 foi de 163, e nos dois triênios sucessivos o número de egressos cresceu mais que 3 vezes (Gráfico 1). 
Apesar do grande crescimento da oferta de cursos de MPs, é possível inferir que o número ainda é insuficiente para atender à demanda dos profissionais que buscam qualificação na área (VILELA RB, BATISTA NA, 2015) (Gráfico 1).

\section{CONSIDERAÇÕES FINAIS}

O presente estudo teve como foco a modesta pretensão de verificar a distribuição e características dos MP em Ensino em Saúde no Brasil. Com base nas informações obtidas nesta pesquisa, foram encontrados 13 MP em ensino em saúde no território nacional em funcionamento avaliados na grande área da Capes Ensino. Foi possível observar que houve avanço nos últimos anos em relação implantação de Mestrados em Ensino em Saúde no Brasil e que esses PPG realmente transformam o local ou objeto de vínculo profissional do aluno. Sobre os programas analisados notou-se a predominância dos vinculados à Instituições Públicas avaliados com nota 3. Do total de 13 PPG, as regiões nordeste e sudeste estão contempladas cada uma com 4 programas, seguidos pelo Centro-Oeste e Norte com 2 programas por região e o Sul com 1 programa. Esta pesquisa assume suas possibilidades e limitações. Aprofundar o tema deve ser a busca constante dos pesquisadores.

\section{REFERÊNCIAS}

1. BARBOSA V. A gênese dos mestrados profissionais em educação nas universidades brasileiras. Plurais, 2016;1(2):94-113.

2. BRASIL. Conselho Federal de Educação. Parecer de no 977/65: Definição dos cursos de pós-graduação. 1965. Disponível em: <www.scielo.br>Acesso em: 23 de mar. 2017.

3. BRASIL. MEC. CAPES. Documento de área: ensino 2016.Disponível em: < www.capes.gov.br>. Acesso em: 11 ago. 2017.

4. BRASIL. MEC. CAPES. Portaria Normativa no 83 de 06 de junho de 2011. São Paulo, 2011. Disponível em: < www.capes.gov.br>. Acesso em: 11 ago. 2017.

5. BRASIL. MEC. CAPES. Diário Oficial da União. Portaria Normativa no 17 de 28 de dezembro de 2009. Disponível em: < www.capes.gov.br>. Acesso em: 11 ago. 2017.

6. BRASIL. MEC. CAPES. Diário Oficial da União.Portaria nํ 389, de 23 de março de 2017. Brasília: DF, 2017. Disponível em: < www.capes.gov.br>. Acesso em: 11 ago. 2017.

7. BRASIL. MEC. CAPES.Diário Oficial da União. Portaria Normativa no 80 de 16 de dezembro de 1998. Disponível em: < www.capes.gov.br>. Acesso em: 11 ago. 2017.

8. GOUVÊA MV, SOUZA ÂC, QUELUCI GC et al. Formação e Educação Permanente em Saúde: processos e produtos no âmbio do Mestrado Profissional. 1ed. São Paulo: Hucitec, 2016; 201p.

9. HORTALE VA, LEAL MC, MOREIRA CO et. al. Características e limites do mestrado profissional na área da Saúde: estudo com egressos da Fundação Oswaldo Cruz. Ciência \& Saúde Coletiva, 2010; 15(4):2051-8.

10. RAZALI NM, WAH YB. Power comparisons of Shapiro-Wilk, Kolmogorov-Smirnov, Lilliefors and Anderson-Darling tests. Journal of Statistical Modeling and Analytics, 2011;2: 21-33.

11. RIBEIRO RJ. Ainda sobre o Mestrado Profissional. Revista Brasileira de Pós-Graduação, 2006;03(06): 313-315.

12. VILELA RB, BATISTA NA. Mestrado profissional em ensino na saúde no Brasil: avanços e desafios a partir de políticas indutoras. Revista Brasileira de Pós-Graduação, 2015;12(28): 307-331.

13. VILELA RB, BATISTA NA. Desafios e práticas para os mestrados profissionais em ensino na saúde. Revista fórum identidades, 2016; 22 (22), 159-172. 